\title{
Early Diagnosis of Neuropathy in Individuals with Family History for Diabetes Mellitus Type 2
}

\author{
Hernandez Gómez JF¹,4 Hernández Sierra JF , CastanedoCazares JP1,2, \\ Rodríguez Rivera JG ${ }^{1,3}$ and Rodríguez Leyva $I^{1,4}$ \\ ${ }^{1}$ Medicine Faculty, Mexico \\ ${ }^{2}$ Dermatology Service, Mexico \\ ${ }^{3}$ Endocrinology Service, Mexico \\ ${ }^{4}$ Neurology Service at the Hospital Central, México
}

ISSN: 2637-7748

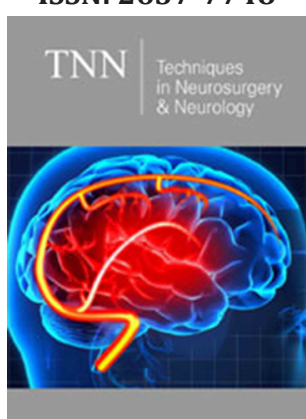

*Corresponding author: Ildefonso Rodriguez-Leyva, Neurology Service at the Hospital Central, México

Submission: 眥 December 1, 2020

Published: 望 December 16, 2020

Volume 3 - Issue 5

How to cite this article: Hernandez Gómez JF, Hernández Sierra JF, Castanedo Cazares JP, Rodríguez Rivera JG, Rodríguez Leyva I. Early Diagnosis of Neuropathy in Individuals with Family History for Diabetes Mellitus Type 2. Tech Neurosurg Neurol. 3(5). TNN. 000571. 2020.

DOI: 10.31031/TNN.2020.03.000571

Copyright@ Ildefonso Rodriguez-Leyva, This article is distributed under the terms of the Creative Commons Attribution 4.0 International License, which permits unrestricted use and redistribution provided that the original author and source are credited.

\begin{abstract}
Introduction: An increase in neuropathy frequency has been observed in individuals with type 2 Diabetes Mellitus (t2DM) and prediabetes, secondary to the associated hyperglycemia. However, the frequency of this pathology in individuals with a family history for t2DM is unknown.
\end{abstract}

Objective: To make a comparison, the frequency of peripheral neuropathy in no diabetic individuals with and without family history for t2DM, and patients with t2DM, evaluated by plantar thermography (PT).

Material and Methods: A pilot, cross-sectional study. One hundred participants were divided into three study groups (control subjects without t2DM and no family history of diabetes, not diabetic subjects with a family history for diabetes and patients diagnosed with diabetes), ANOVA, and multiple logistic regression were made to analyze the outcomes.

Result: Sixty-four subjects with altered PT were found, of which 26 belonged to the group of diabetics, 23 to the non-diabetics with family history for t2DM, and 15 to the group, not 2tDM without a family history ( $p=0.003$ ). In diabetic patients, neuropathy was $76.4 \%$, non-diabetics subjects with family history $67.6 \%$, and controls of $46.8 \%$, with OR of 3.6, 2.3, and 1.0 respectively, and a p of 0.001 in a multivariate analysis.

Conclusion: The presence of neuropathy in diabetic patients is independent of age, sex, and hypertension. A tendency to present neuropathy diagnosed by thermography was observed by having at least one family member in the first degree with $\mathrm{t} 2 \mathrm{DM}$, with an association between the number of first-degree relatives and the problem's occurrence. Prevention measures must apply as prevention of neuropathy and t2DM when family history is present

Keywords: Alzheimer's; Parkinson's; Cerebrospinal Fluid; Laboratory Diagnosis

\section{Background}

Diabetes mellitus type 2 is a chronic degenerative disease whose impact on older adults' morbidity and mortality has made it one of the leading public health problems [1]. In Mexico, $10 \%$ of the population is diabetic, almost half of them suffer systemic arterial hypertension (ASH), and most have a family history of diabetes [2]. Polyneuropathy has been reported from 8 to $54 \%$ in type 1 diabetic patients and 13 to $46 \%$ in type 2 diabetic patients [3-6]. Besides, its frequency is higher in individuals with impaired glucose tolerance (IGT) compared with those who have normal glucose tolerance (NGT) [7]. It has even shown that it can start even before prediabetes features [8], implying alterations in the metabolism of polyols in nerve cells secondary to subtle changes in glycemia [9]. A usual evaluation method for diagnosing neuropathy is the MNSI (Michigan Neuropathy Screening Instrument) [10].

This procedure includes first inspecting the feet, evaluating deformities, dry skin, calluses, and infection, besides the presence of ulcers and loss of myotatic reflexes. Despite nerve conduction velocity, which is considered the most convenient diagnostic method and the skin biopsy considered the gold standard, are both invasive and painful to access; the first is especially useful for large nerve fibers, and the second requires an expert to evaluate. However, the intra- and interobserver variability of the MNSI and the lateness of the diagnosis has forced to seek new and less painful diagnostic alternatives. Plantar thermography 
(TP) has become an attractive alternative, has a sensitivity of $81.3 \%$ [11], and reproducibility of $95 \%$ to $100 \%$ [12] in diabetic patients. The diabetic neuropathies can be divided into a) possible (symptoms or signs), b) probable (symptoms and signs with two of the following: reduced distal sensation, diminution or absent distal myotatic reflexes, c) confirmed: symptoms or signs with abnormal and compatible neuro-conduction studies (NCS) or validated small fiber measure, d) subclinical: abnormal NCS or validated small fiber measure without clinical manifestations [13]. The present study aims to determine if there are reproducible thermographic data that suggest diabetic neuropathy in individuals with a family history without clinical or laboratory manifestations of t2DM, which allows it to propose this technique as an early detection method to evaluate this complication early of type 2 diabetes mellitus.

\section{Material and Methods}

\section{Study design}

Prospective analytical cross-sectional study. Individuals older than 18 years of age of either sex included in three groups:

a) Individuals without diabetes mellitus and a family history for $\mathrm{t} 2 \mathrm{DM}$

b) Individuals without diabetes mellitus, with a family history for $\mathrm{t} 2 \mathrm{DM}$

\section{c) Patients diagnosed with t2DM}

They were sampled by convenience from the endocrinology and neurology services between October 2016 and April 2017. Those with autoimmune diseases or Raynaud's "phenomenon," peripheral vascular insufficiency, lower limb trauma in the last three months, disc herniation, infection of the skin or soft tissues of the lower limbs, clinical data of vasculitis, edema of the lower limbs, recent antimicrobial treatments, and type 1 diabetes mellitus were excluded. The protocol was authorized by the ethics and research committee of the Hospital Central "Dr. Ignacio Morones Prieto" from San Luis Potosi, Mexico, and registered with the number 32-16.

\section{Subjects}

The participants were allocated in one of three different study groups: a) without diabetes mellitus, and no family history for t2DM demonstrated at interrogation and with biochemical evidence of fasting blood glucose less than $100 \mathrm{mg} / \mathrm{dl}$; b) without diabetes mellitus, with a family history for t2DM, demonstrated by interrogation and with biochemical evidence of fasting blood glucose less than $100 \mathrm{mg} / \mathrm{dl}$. Family history for t2DM in at least one first-degree relative diagnosed and undergoing t2DM treatment at interrogation, c) Patients with a diagnosis of t2DM demonstrated with biochemical evidence of fasting blood glucose greater than $125 \mathrm{mg} / \mathrm{dl}$, glycosylated hemoglobin more significant than 6.5\%, glycemia greater than $200 \mathrm{mg} / \mathrm{dl}$ in a random sample or two hours after a load of $75 \mathrm{~g}$ of oral glucose, or history of a self-recognized disease treated with hypoglycemic agents or insulin.

\section{Thermography}

Peripheral neuropathy was diagnosed by thermography according to the guidelines of the American Thermography Association [14]. They included the Thermal recovery index (TRI), calculated as the ratio between the average plantar temperature of 10 sites of interest (both heels, upper toes, and heads) metatarsophalangeal 1,3 , and five at rest and after exposure to water at 15 degrees centigrade for one minute. A ratio of $<90 \%$ or $>$ $100 \%$ is considered abnormal for this test. The Interdigital thermal gradient (anisothermal) was also evaluated. It was positive if it was higher than 0.4 degrees centigrade 10 minutes after exposure for 1 minute to water at 15 degrees centigrade. The procedure consisted of taking a basal thermal photograph of the individuals' feet' soles after 15 minutes' acclimatization at room temperature. Next, the subjects' feet were covered with a plastic bag to avoid contact with the water and were submerged in the water at 15 degrees centigrade for 60 seconds. Another thermal image was taken 10 minutes after removing the feet from the water. These images were obtained using the Infrared Camera Flir model 7i (Wilsonville, Oregon, USA) and evaluated utilizing a specialized software for obtaining an average of the temperature from 10 areas of interest in the feet (FLIR Tools Software 5.0 version). The Infrared Camera was an uncooled IR imager with a Focal Plane Array of VanadiumOxide microbolometers; it has a thermal sensitivity better than 0.1 ${ }^{\circ} \mathrm{C}$ at $25^{\circ} \mathrm{C}$, a field of view of $29^{\circ} \times 29^{\circ}, 60 \mathrm{~cm}$ focus, and a spectral range from 7.5 to $13 \mathrm{~mm}$. The camera provided individuals' thermal information in real-time, with a 140x140 pixel resolution saved in digital format for further processing; the emissivity was set at 0.97 for all the measurements.

\section{Statistical Analysis}

The sample size of 30 individuals per group was considered expecting a difference of 0.40 between healthy without load t2DM and individuals with $\mathrm{t} 2 \mathrm{DM}$ with $95 \%$ confidence and power of $80 \%$ two tails. The analysis of normality was made with the ShapiroWilk test, the comparison between groups was by Chi-square (Mantel and Haenzell) for nominal variables, and by ANOVA test for continuous variables. The rest of the data we analyzed by multiple logistic regression [15]. We considered the presence of polyneuropathy by thermography, the dependent variable, and as independent variables, the presence or absence of family history for t2DM and t2DM. Factors of confusion were age, sex, time of evolution, and ASH. The statistical analysis was carried out with $\mathrm{R}$, version 3.4.1 software (R Foundation for Statistical Computing). All $\mathrm{P}$ values were for a 2-sided test, and results were considered statistically significant at $<0.05$. The interobserver kappa value for the diagnosis of neuropathy by thermography was 0.9 .

\section{Result}

A total of 100 patients were included, 34 in the group with diabetes mellitus, 34 in the apparent health group with a family history for $\mathrm{t} 2 \mathrm{DM}$, and 32 from the apparent health group without 
such family history. The ages ranged between 23 and 85 years (mean 55 years, $\mathrm{SD}=11.3$ years), with a predominance of the female sex in $77 \%$. The mean evolution time was $14.1+10.3$ years, and the prevalence of ASH was $43 \%$. The average age found for the group with abnormal TRI was 56.6+11.1 years and was not different from the group with normal TRI having 52.0+11.9 years. Regarding sex, $76.6 \%$ of patients with altered TIR were women, a similar proportion found in the group with typical values (77.7\%). On the other hand, the ASH frequency was $53 \%$ in the group of abnormal TRIS, which was almost twice higher than patients with normal TRI (25\%). The group affected by diabetes exhibited an abnormal TRI in $76.4 \%$ (26/34); this was different from subjects without a family history who has had it abnormal in 46.8\% (15/32), (OR=0.2, CI95 0.09-0.7), $\mathrm{P}=0.01)$. The group healthy with family history showed an abnormal test of $67.6 \%(23 / 34)$ and was not different from the diabetic group $(\mathrm{P}=0.09)$. Following the diagnosis of neuropathy by thermography, it was found that $87.5 \%$ of them had clinical symptoms such as numbness, tingling, pain, the experience of a sensation that shoes not fitting well or the socks being bunched up, burning, electric and stabbing sensations or weakness, especially with a glove and sock distribution. Interestingly, we found a more significant number of first-degree relatives affected by t2DM among those healthy individuals with an abnormal TRI test, compared with average results ( 2.7 vs. 0.9 relatives, U Mann-Whitney, $\mathrm{P}=$ $0.004)$. The $O R$ between $2 \mathrm{DM}$ and the control group shows a value of 3.7 with a probability of $70 \%$ finding neuropathy (Tables 1 \& 2). The OR comparing the groups without $\mathrm{t} 2 \mathrm{DM}$ with family history was 2.4 , with the possibility of having $70 \%$ more positivity over the group without t2DM neither family history (Figures 1-3).

Table 1: Confounding variables in patients with diabetes and subjects with and without a family history for type 2 diabetes mellitus.

P-Results from the variance analysis for age and diagnostic time; and Chi-squared test for sex, arterial hypertension, and neuropathy symptoms. NS: No, significative.

\begin{tabular}{|c|c|c|c|c|}
\hline \multirow{3}{*}{ Variable } & \multicolumn{3}{|c|}{ Group } & \multirow{3}{*}{$\mathbf{P}$} \\
\hline & Diabetic Population & Subjects with diabetic charge & Subjects without family history & \\
\hline & $\mathrm{N}=34$ & $\mathrm{~N}=34$ & $\mathrm{~N}=32$ & \\
\hline Age(years) & $58.7+11.5$ & $50+8.6$ & $56.2+12.8$ & $P=0.005$ \\
\hline Sex(female) & 27 & 26 & 24 & NS \\
\hline Arterial hypertension & 23 & 9 & 11 & $\mathrm{P}=0.001$ \\
\hline Diagnostic time (years) & $14.1+10.3$ & 0 & 0 & $\mathrm{P}=<0.001$ \\
\hline Symptoms of neuropathy & 23 & 10 & 2 & $\mathrm{P}=<0.001$ \\
\hline
\end{tabular}

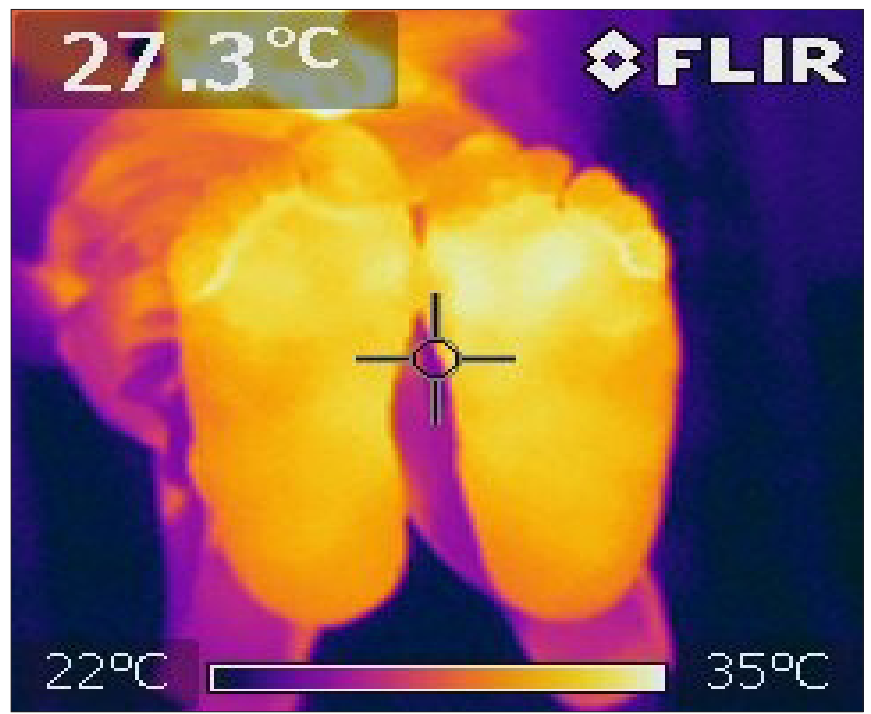

Figure 1: Subjects without family history for t2DM. A high and homogeneous level of color and temperature in the distal part of the feet is observed.

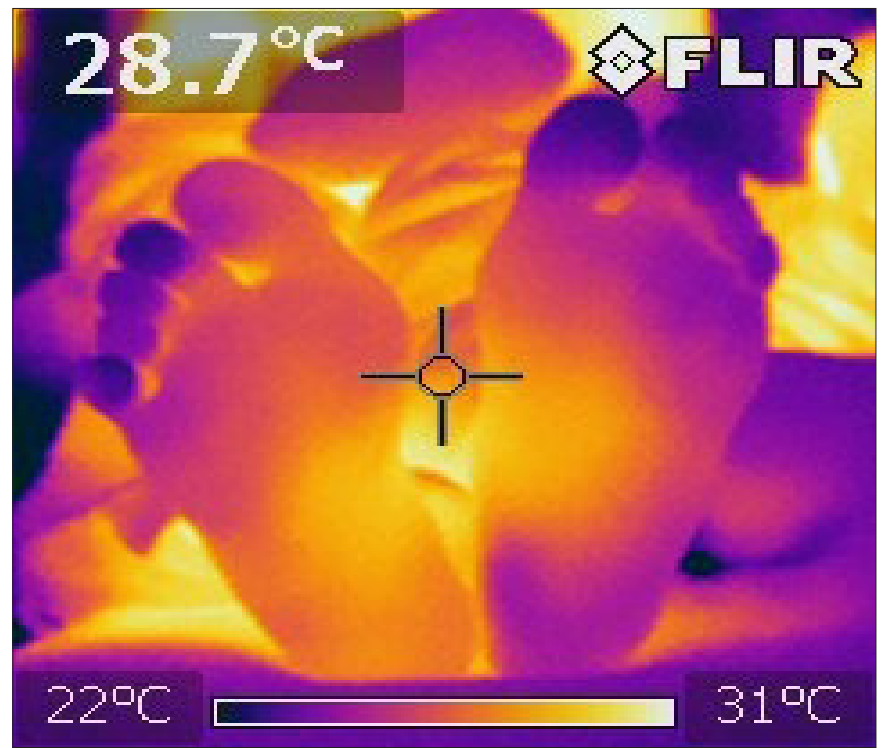

Figure 2: Subject with family history for t2DM.A heterogeneous color and temperature in the distal aspects of the feet are observed in the image. 


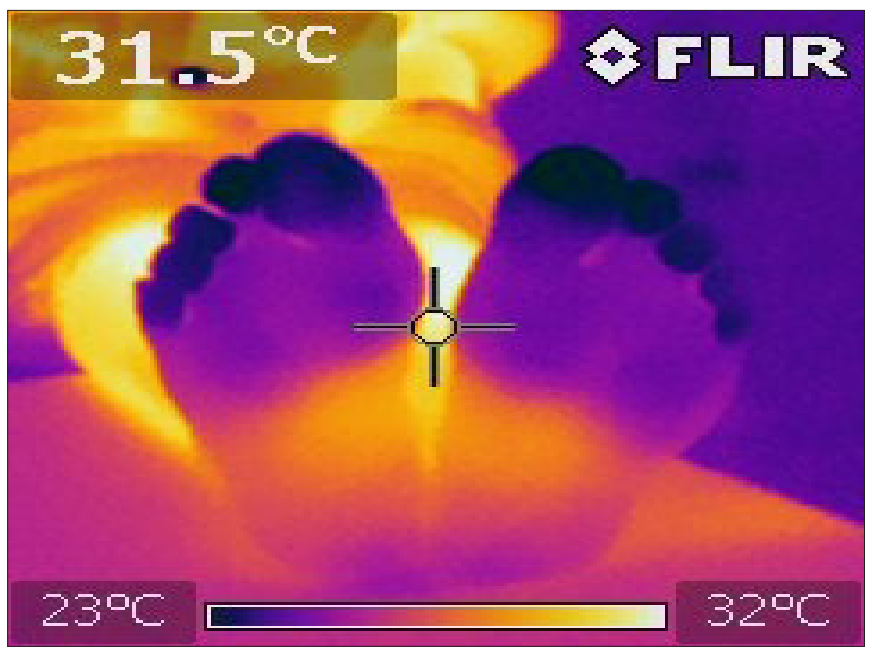

Figure 3: Affected neuropathic patients with t2DM. A heterogeneous pattern of low temperature is observed on the distal part of the feet, which contrasts with a higher temperature in the legs' proximal part.

Table 2: An OR of 2.3 with the antecedent $t 2 D M$ in the family, and the OR 3.6 if the subject is a diabetic person.

\begin{tabular}{|c|c|c|c|}
\hline \multicolumn{4}{|c|}{ Relation Antecedents HF-DM vs Controls } \\
\hline & Cases & Controls & Total \\
\hline Expuesto & 23 & 11 & 34 \\
\hline NoExp & 15 & 17 & 32 \\
\hline Total & 38 & 28 & 66 \\
\hline \%FrecExp & 60.53 & 39.29 & 51.52 \\
\hline$X^{2}=$ & 2.912 & $\mathrm{OR}=$ & 2.37 \\
\hline $\mathrm{P}=$ & 0.088 & $\mathrm{ORi}=$ & 0.88 \\
\hline ORs $=$ & 6.38 & RAP $\%$ & \\
\hline RAP (FracEt) & 0.35 & 35 & \\
\hline $\mathrm{RAP}_{\mathrm{i}}=$ & 0.112 & 11.2 & \\
\hline $\mathrm{RAP}_{\mathrm{s}}=$ & 0.524 & 52.4 & \\
\hline \multicolumn{4}{|c|}{ Relation DM vs Controls } \\
\hline & Cases & Controls & Total \\
\hline DM & 26 & 8 & 34 \\
\hline NoExp & 15 & 17 & 32 \\
\hline Total & 41 & 25 & 66 \\
\hline \%FrecExp & 63.41 & 32 & 51.52 \\
\hline$X^{2}=$ & 6.136 & $\mathrm{OR}=$ & 3.68 \\
\hline $\mathrm{P}=$ & 0.013 & $\mathrm{ORi}=$ & 1.31 \\
\hline ORs $=$ & 10.33 & RAP \% & \\
\hline RAP(FracEt) & 0.462 & 46.2 & \\
\hline $\mathrm{RAP}_{\mathrm{i}}=$ & 0.253 & 25.3 & \\
\hline $\mathrm{RAP}_{\mathrm{s}}=$ & 0.613 & 61.3 & \\
\hline
\end{tabular}

\section{Discussion}

We use thermography to demonstrate subjacent damage to peripheral nerves in subjects with and without family burden for t2DM and t2DM since we have found in our clinical practice that relatives and diabetics have a higher incidence of this condition. It is recognized that diabetic neuropathy is an early complication of diabetes and that it can manifest itself before the presence of diabetes, usually with a more distal than proximal condition [16]. PT gives us the great advantage of detecting the condition of large and small fibers [17]; it is also harmless and free of complications. The procedure is not painful and has a relatively low cost compared to other techniques. In this study, we observed a lower risk of polyneuropathy when we compared subjects with no family history concerning people with diabetes. A strong association was found between the number of first-degree relatives with DM2 and the neuropathy diagnosed by thermography, reflecting the importance of family background for t2DM and the risk of complications. Family history may be related to different polymorphisms, such as the angiotensin-converting enzyme, but more than five hundred gene expression profiles are associated with diabetic neuropathy [18]. All of them can play an important role not only for the presence of multiple factors, like dyslipidemia (increase triglycerides, LDL, FFA, with diminution of HDL), DNA damage, ER stress, mitochondrial dysfunction, cellular injury, and irreversible damage of the peripheral nerves.

Besides, HSA but is also directly associated with microvascular ischemia that affects the nerves, which explains the neuropathic problem $[19,20]$. Neuropathy has been related to age, male gender, and low glycemic level control [21,22]. However, in this study, neuropathy was independent of age, sex, and arterial hypertension. We propose thermography as a screening test that can be used in the future to detect early neuropathy in DMT2, as well as for the detection of subjects at risk, especially in those with a family history, since the use of metformin, statins, and a substantial intervention in the lifestyle can change the natural history of the disease and especially practice exercise [23-26]. Thermography can be performed in endocrinology services worldwide, considering that it is non-expensive and easily accessible. A positive result could force an in-depth analysis of this dysfunction in the affected subject, beginning with a targeted interrogation, a complete physical examination, and conducting electrophysiology studies, which unfortunately only detect the condition of large fibers [27]. It is forced to diagnose early in subjects at risk or with positive studies because diabetic neuropathy removes quality and shortens the sufferer's life. However, further studies will be necessary with larger groups to detect if other involved risk factors such as smoking or sedentary life [28], also will be necessary to include comparable subjects similar in age and sex and other demographic, environmental, and familial characteristics.

\section{Conclusion}

There is a high risk of suffering from neuropathy with the family charge of diabetic in the family. Still, other factors can be 
involved, but there is a clear difference in the problem's frequency in subjects without diabetic genetic charge and this antecedent. The thermography can be a useful method to detect this possible diagnosis in people with risk factors.

\section{References}

1. Sarah W (2004) Global prevalence of diabetes: Estimates for the year 2000 and projections for 2030. Diabetes Care 27(5): 1047-1053.

2. Hernández Á, Mauricio J, Pablo G, Nancy RN (2013) Diabetes mellitus en México: El estado de la epidemia. Salud Pública Méx 55(2): 129-136.

3. Donald (2000) The incidence and lifetime prevalence of neurological disorders in a prospective community-based study in the UK. Brain 123(4): 665-676.

4. Knuiman (1986) Prevalence of diabetic complications in relation to risk factors. Diabetes 35(12): 1332-1339.

5. Fujimoto, Wilfred Y (1987) Prevalence of complications among secondgeneration Japanese American men with diabetes, impaired glucose tolerance, or normal glucose tolerance. Diabetes 36(6): 730-739.

6. Dyck, Peter J (1993) The prevalence by staged severity of various types of diabetic neuropathy, retinopathy, and nephropathy in a populationbased cohort: the rochester diabetic neuropathy study. Neurology 43(4): 817-817.

7. Wong, Tien Y (2005) Retinopathy in persons with impaired glucose metabolism: the Australian diabetes obesity and lifestyle (AusDiab) study. American Journal of Ophthalmology 140(6): 1157-1159.

8. Diabetes Prevention Program Research Group (2007) The prevalence of retinopathy in impaired glucose tolerance and recent-onset diabetes in the diabetes prevention program. Diabetic Medicine 24(2): 137-144.

9. Abuzinadah, Ahmad R (2017) Less is more in diabetic neuropathy diagnosis: comparison of quantitative sudomotor axon reflex and skin biopsy. Journal of Clinical Neuromuscular Disease 19(1): 5-11.

10. Feldman, Eva L (1994) A practical two-step quantitative clinical and electrophysiological assessment for the diagnosis and staging of diabetic neuropathy. Diabetes Care 17(11): 1281-1289.

11. Balbinot, Luciane F (2012) Plantar thermography is useful in the early diagnosis of diabetic neuropathy. Clinics 67(12): 1419-1425.

12. Balbinot, Luciane Fachin (2013) Repeatability of infrared plantar thermography in diabetes patients: a pilot study. J Diabetes Sci Technol 7(5): 1130-1137.

13. Tesfaye, Solomon (2010) Diabetic neuropathies: update on definitions, diagnostic criteria, estimation of severity, and treatments. Diabetes Care 33(10): 2285-2293.
14. Bharara, Manish, Cobb JE, Claremont DJ (2006) Thermography and thermometry in the assessment of diabetic neuropathic foot: a case for furthering the role of thermal techniques. Int J Low Extrem Wounds 5(4): 250-260.

15. Feinstein, Alvan R (1996) Multivariable analysis: an introduction. Yale University Press, London, UK.

16. Fraser DM (1977) Peripheral and autonomic nerve function in newly diagnosed diabetes mellitus. Diabetes 26(6): 546-550.

17. Guy RJC (1985) Evaluation of thermal and vibration sensation in diabetic neuropathy. Diabetologia 28(3): 131-137.

18. Hur, Junguk (2011) The identification of gene expression profiles associated with progression of human diabetic neuropathy. Brain 134(11): 3222-3235.

19. Pop Busui, Rodica (2017) Diabetic neuropathy: a position statement by the American diabetes association. Diabetes Care 40(1): 136-154.

20. Moleda P (2007) Relationship between I/D polymorphism of angiotensin i converting enzyme gene and microvascular complications in type 2 diabetic patients. Przegl Lek 64(3): 134-139.

21. DCCT Research Group (1988) Factors in development of diabetic neuropathy: baseline analysis of neuropathy in the feasibility phase of diabetes control and complications trial (DCCT). Diabetes 37(4): 476481.

22. Arezzo, Joseph C (1999) New developments in the diagnosis of diabetic neuropathy. The American Journal of Medicine 107(2): 9-16.

23. Herman, William H (2005) The cost-effectiveness of lifestyle modification or metformin in preventing type 2 diabetes in adults with impaired glucose tolerance. Ann Intern Med 142(5): 323-332.

24. Connor, Patrick J, Stephen JS, Steven HW (1998) Care of adults with type 2 diabetes mellitus. a review of the evidence. The J fam Pract 47(5): S1322.

25. Perreault, Leigh (2012) Effect of regression from prediabetes to normal glucose regulation on long-term reduction in diabetes risk: results from the diabetes prevention program outcomes study. Lancet 379(9833): 2243-2251.

26. Singleton, Robinson J (2015) Supervised exercise improves cutaneous reinnervation capacity in metabolic syndrome patients. Ann Neurol 77(1): 146-153.

27. Kimura, Jun, Thoru Y, Nelson P (1979) Distal slowing of motor nerve conduction velocity in diabetic polyneuropathy. Journal of the Neurological Sciences 42(2): 291-302.

28. Mitchell, Braxton D, Victor MH, Aaron IV (1990) Cigarette smoking and neuropathy in diabetic patients. Diabetes Care 13(4): 434-437. 